\title{
“Paper Machine” for Molecular Diagnostics
}

\section{Citation}

Connelly, John T., Jason P. Rolland, and George M. Whitesides. 2015. “'Paper Machine' for Molecular Diagnostics." Anal. Chem. 87 (15) (August 4): 7595-7601. doi:10.1021/ acs.analchem.5b00411.

\section{Published Version}

doi:10.1021/acs.analchem.5b00411

\section{Permanent link}

http://nrs.harvard.edu/urn-3:HUL.InstRepos:24900309

\section{Terms of Use}

This article was downloaded from Harvard University's DASH repository, and is made available under the terms and conditions applicable to Open Access Policy Articles, as set forth at http:// nrs.harvard.edu/urn-3:HUL.InstRepos:dash.current.terms-of-use\#OAP

\section{Share Your Story}

The Harvard community has made this article openly available.

Please share how this access benefits you. Submit a story.

\section{Accessibility}




\title{
A "Paper Machine" for Molecular Diagnostics
}

\author{
John T. Connelly ${ }^{\mathrm{a},}{ }^{*}$, Jason P. Rolland ${ }^{\mathrm{a}}$, and George M. Whitesides ${ }^{\mathrm{b}}$ \\ ${ }^{a}$ Diagnostics For All, 840 Memorial Drive, Cambridge, MA 02139 \\ ${ }^{\mathrm{b}}$ Department of Chemistry and Chemical Biology, Harvard University, Cambridge MA \\ 02138 \\ *Email: jconnelly@dfa.org Tel: +1 6174940700
}

\begin{abstract}
Clinical tests based on primer-initiated amplification of specific nucleic acid sequences achieve high levels of sensitivity and specificity. Despite these desirable characteristics, these tests have not reached their full potential because their complexity and expense limit their usefulness to centralized laboratories. This paper describes a device that integrates sample preparation and Loop-mediated Isothermal Amplification (LAMP) with endpoint detection using a handheld UV source and camera phone. The prototype device integrates paper microfluidics (to enable fluid handling) and a multi-layer structure - or a "paper machine" - that allows a central patterned paper strip to slide in and out of fluidic path, and thus to allow introduction of sample, wash buffers, amplification master mix, and detection reagents with minimal pipetting, in a hand-held, disposable device intended for point-of-care use in resource-limited environments. This device creates a dynamic seal that prevents evaporation during incubation at $65^{\circ} \mathrm{C}$ for 1 hour. This interval is sufficient to allow a LAMP reaction for the Escherichia coli malB gene to proceed with an analytical sensitivity of 1 target copy. Starting with human plasma spiked with whole,
\end{abstract}


live $E$. coli cells, this paper demonstrates full integration of sample preparation with LAMP amplification and endpoint detection with a limit of detection of 5 cells. Further, it shows that the method used to prepare sample enables concentration of DNA from sample volumes commonly available from fingerstick blood draw.

\section{Introduction}

The unparalleled sensitivity and specificity of nucleic acid amplification tests (NAATs), led by those based on polymerase chain reaction (PCR), have become increasingly available in the developed world for the diagnosis of infectious, genetic, and other diseases. In developing countries these tests are much less used because of their cost, complexity and requirements for expensive equipment and reagents. PCR requires three precisely controlled temperatures to enable melting of target DNA, annealing, and elongation of primers, and is typically carried out using expensive thermocyclers. Additionally, prior to amplification NAATs usually require sample preparation involving a series of steps to release the DNA, and to remove the many inhibitory compounds found in clinical samples ${ }^{1,2}$. These steps often require the use of complex laboratory equipment and, therefore, highly trained personnel. This combination of factors expensive and delicate equipment, trained personnel, and complex procedures - has kept NAATs from being broadly adopted in resource-limited countries (although they are, of course, used to limited extents in centralized hospitals and government laboratories). Because transporting patients and/or samples to these central facilities can be difficult, slow, and expensive in resource-limited environments with under-developed transportation systems, local healthcare outposts and traveling healthcare workers are the often the primary, or only, providers of healthcare ${ }^{3-6}$. 
NAATs intended for use in resource-limited settings must operate within the constraints of those environments. One diagnostic technology that has been successfully implemented at the point of care is lateral flow assays, particularly lateral flow immunochromatographic strips ${ }^{6}$. These systems require minimal sample preparation and no instrumentation. They typically provide a visual result within 10 to 20 minutes $^{6}$. Tests for direct or indirect detection of a range of pathogens (including Trypanosoma cruzi $^{7}$, diphtheria toxin ${ }^{8}$, Plasmodium falciparum $^{9}$, and Haemophilis ducreyi ${ }^{10}$ ) have been reported, tested, and are available in resource-limited settings. Lateral flow assays have also been used for endpoint detection of PCR and isothermal amplification products, either from reactions conducted using standard equipment ${ }^{11-14}$ or integrated into microfluidic systems ${ }^{15-17}$. Though lateral flow endpoint detection can simplify the final readout of NAATs, the instrumentation required remain (often prohibitively) complicating factors, from sample preparation through amplification, and which may include open-channel microfluidic systems with external pumps.

Despite the obstacles to implementation of NAATs at the point of care, a number of systems are being developed for this type of application. The Cepheid GeneXpert (Sunnyvale, CA) is a notable current example. The GeneXpert reduces user handling of samples by integrating sample preparation steps with PCR amplification and real-time fluorescence detection in the same cartridge ${ }^{18}$. Though meeting the ideal of a sample-in, answer-out device, the GeneXpert has complex optical and electronic systems required for real-time PCR, and pumps to move sample and reagents through the cartridge, and is still not a general solution for resourcelimited environments ${ }^{19}$.

We have chosen to use isothermal amplification methods in the system described here. These methods simplify molecular diagnostics by eliminating thermocycling, reducing 
equipment costs and power requirements, without sacrificing sensitivity or specificity. In particular, Loop-mediated Isothermal Amplification (LAMP) $)^{20,21}$ has a high tolerance for common components present in clinical samples that inhibit PCR and other reactions ${ }^{22-25}$. This robust tolerance toward inhibitors further streamlines assays, because it simplifies sample preparation and complexity/expense of equipment ${ }^{26}$. Although devices integrating LAMP amplification with detection have entered the market (for example, the IllumiGene ${ }^{\circledR}$ system; Meridian Bioscience, Cincinnati, $\mathrm{OH})$, they have not directly incorporated the steps necessary for sample preparation into the instrument ${ }^{27}$. Further, as products targeted toward developed world settings, they employ complex optical systems and user interfaces that increase cost and, especially, the requirements for maintenance and repair.

By merging the useful characteristics of lateral-flow assays with those of open-channel microfluidic systems, paper microfluidic platforms using three-dimensional stacked patterned paper $^{28-31}$ or two-dimensional networks of cut paper $^{32-34}$ allow complex fluid handling without the need for external pumps and power. Paper as a substrate for diagnostics has other beneficial properties as well: (i) It is biocompatible (and has been used for decades for storage of clinical samples in the form of dried blood spots, and is also widely used as a chromatography medium); (ii) Because DNA adsorbs to cellulose ${ }^{35}$, paper provides a simple matrix for its capture and isolation; (iii) The cellulose fiber network provides a high surface area, and facilitates storage of dried reagents $^{36}$. A paper-based test for liver function (Diagnostics For All) has been successful in field trials; this test provides semi-quantitative measurements of liver transaminases from whole blood ${ }^{37,38}$. Despite these advances, there have, however, been no demonstrations of NAATs in complete systems (blood to read-out) in paper microfluidic systems. 
In important steps toward the objective of a complete system, Rohrman and RichardsKortum have demonstrated Recombinase Polymerase Amplification (RPA) in a paper-based device (using sample preparation and detection as separate steps) ${ }^{39}$. The Ellington group demonstrated detection of LAMP products in paper microfluidic devices using their Catalytic Hairpin Assembly (CHA) reaction, which uses a series of specially designed oligonucleotide hairpins to specifically identify the target and provide an additional signal enhancement. Here, the amplification reaction was conducted off-chip ${ }^{40}$.

Our goal is to develop a NAAT device that is compact, inexpensive, and fully integrates sample preparation, amplification, and detection in a low-cost, single-use format appropriate for point-of-care diagnostics in resource-limited settings. To this end, we describe a prototype paper microfluidic device that enables sample preparation from clinically-relevant matrices, isothermal amplification, and detection; the cost of materials for the device is US\$ 0.60 , and a total cost per assay of US\$ 1.83 (Table S1). These costs, of course, do not indicate the cost of a fully developed product based on this type of system, but they do indicate that the cost of materials will not limit affordability. Figure 1a shows the multi-layer architecture of device - which we hereafter refer to as a "sliding strip;" this design allows for the middle layer - the strip containing the "reaction vessel," a paper disc in which reaction and washing steps occur, to slide into different fluidic paths. The system is, thus, a "paper machine": that is, a device fabricated in paper, with moving parts, which accomplishes a task.

The linear motion of the sliding strip relative to the stationary surrounding structures, acts as a valve to control the serial introduction of sample, wash buffer, amplification reagents, and detection reagents, while also dynamically sealing to prevent evaporation during incubation steps. The sample preparation steps are simplified by fabricating the reaction disc out of 
Whatman FTA ${ }^{\circledR}$ paper, which contains a proprietary blend of lytic reagents dried into the cellulose matrix. FTA ${ }^{\circledR}$ paper has been shown to lyse a wide range of cells and viruses ${ }^{41-43}$, and, in its normal use, after extensive washing in tubes with vortexing, it can be added directly to PCR reactions. Our device replaces this instrumented, multistage, high-volume washing with two, through-flow washes before the introduction of LAMP master mix. Also, by exploiting the hydrophilicity of paper, we simplify fluid handling by replacing external mechanical systems for pumping with fluid flow driven by capillary action. Final detection of the amplified product uses SYBR Green I, a fluorescent intercalating dye, and a handheld UV source and camera phone. For a proof-of-concept, we used LAMP to amplify a portion of the malB gene common to all Escherichia coli $^{44}$.

\section{Materials \& Methods}

Oligonucleotide primers were ordered from Eurofins Operon MWG (Huntsville, AL) with HPLC purification and suspended in 1X Tris-EDTA. Bst 2.0 DNA polymerase, 10X Isothermal Amplification Buffer, dNTPs, DNA/DNAse - free stocks of $\mathrm{MgSO}_{4}$, and restriction endonucleases were purchased from New England Biolabs (Ipswich, MA) and betaine from Sigma-Aldrich (St. Louis, MO). Pooled human plasma in sodium heparin derived from whole blood donations was acquired from Valley Biomedical Products and Services, Inc. (Winchester, VA). Pre-cast $2 \%$ agarose gels and SYBR Green I were purchased from Invitrogen. All other reagents were supplied by Fisher Scientific.

\section{Fabrication of magnetic sliding-strip devices and confirmation of evaporation-resistance \\ The magnetic material used to fabricate the devices is $0.75 \mathrm{~mm}$ thick synthetic rubber- bonded ferrite magnetic sheeting with $3 \mathrm{~mm}$ bands of alternating poles on both faces obtained}


from McMaster-Carr (Robbinsville, NJ). The magnetic sheets, as well as all laminate layers, were cut using a design file from Adobe Illustrator and a Graphtec CraftRoboPro (Graphtec Irvine, CA) knife plotter. A thin film of Krytox ${ }^{\circledR}$ LVP lubricant (DuPont Wilmington, DE) is applied with a roller to the inner faces of the top and lower layers as well as to both sides of the sliding strip. The reaction disc is $4.75 \mathrm{~mm}$ diameter and consists of Whatman FTA ${ }^{\circledR}$ paper while the wash vias are $6.35 \mathrm{~mm}$ diameter discs cut from Ahlstromm 226 paper. The various layer were stacked, laminated, and pressed as illustrated in Figure 1a.

The attractive force produced by the magnetic material forms an evaporation-resistant seal in combination with the thin film of lubricant applied to the layer interfaces. This seal was tested by applying $10 \mu \mathrm{L}$ of water to the paper reaction disc within a sliding-strip device, which was pre-dried to remove any ambient moisture, and slid to seal. The mass of the device before and after the addition of the water was recorded to serve as the baseline. This device was then incubated at $65^{\circ} \mathrm{C}$ for 1 hour, weighed, and slid to unseal the disc. The device was returned to the incubator to completely evaporate any water remaining and the final mass recorded. The masses of the device after incubation and after complete evaporation were used to determine the amount of water lost during incubation by comparison to the baseline.

\section{E. coli Culture}

We used E. coli BL21(DE3)pLysS as a model organism in this work. Cells were cultured overnight at $37^{\circ} \mathrm{C}$, shaking, from frozen glycerol stocks in LB broth containing $34 \mu \mathrm{g} / \mathrm{mL}$ chloramphenicol. We determined the concentration of cells by measuring the optical density at $600 \mathrm{~nm}$ and comparing this value to a growth curve. When directly used to spike sample, the culture was centrifuged, the supernatant aspirated, and the pellet resuspended to the desired 
concentration with fresh media, twice, to remove any free DNA. This stock of cells was then serially diluted in human plasma for experiments.

\section{LAMP Amplification}

The primer set (Table S2) targeting the malB gene of E. coli, including loop primers, as described by Hill et al. were employed in this work ${ }^{44}$. LAMP was performed using the $B s t 2.0$ DNA Polymerase, an in silico designed homologue of Bst DNA Polymerase I, Large Fragment, commonly used in this reaction, and its supplied buffer ${ }^{45}$. In transitioning the reaction from tubes to paper, the concentration of betaine, primers, and enzyme were optimized, as was the incubation time, to minimize non-specific reactions and maximize sensitivity, as shown in Figure $\mathrm{S} 1$ and discussed in the supplementary information. The final reactions contained $1 \mathrm{X}$ Isothermal Amplification Buffer (10 mM Tris-HCl, 10 mM (NH$)_{2} \mathrm{SO}_{4}, 50 \mathrm{mM} \mathrm{KCl,} 2 \mathrm{mM} \mathrm{MgSO}$, 0.1\% Tween-20) supplemented with an additional $6 \mathrm{mM} \mathrm{MgSO}_{4}, 0.9 \mathrm{M}$ betaine, $1.4 \mathrm{mM}$ each dNTP, $1.6 \mu \mathrm{M}$ each inner primer (FIP, BIP), $0.8 \mu \mathrm{M}$ each loop primer (LF, LB), $0.2 \mu \mathrm{M}$ each outer primer $(\mathrm{F} 3, \mathrm{~B} 3)$, and $0.32 \mathrm{U}$ of polymerase. The optimal incubation was $65^{\circ} \mathrm{C}$ for $60 \mathrm{~min}$. The reaction was always run with two negative controls - a no-template control (NTC) and a noamplification control (NA+), containing a heat-inactivated enzyme and 1,000 copies of target or 1,000 cells, as appropriate. Reactions in tubes were carried out at $25 \mu \mathrm{L}$; in paper, the reaction volume was $10 \mu \mathrm{L}$.

\section{Product Analysis by Gel Electrophoresis}

To confirm that positive signals were the result of specific, template-driven reactions, LAMP products were analyzed by agarose gel electrophoresis (AGE). First, a positive reaction conducted in a tube using 1,000 copies of the target was digested using restriction endonuclease 
$P v u I I$, which recognizes a sequence in region $\mathrm{F} 1$ of the target, and run on a $2 \%$ agarose gel with a DNA ladder and the un-digested product to determine if the observed LAMP banding pattern was the result of template-specific amplification and to mark said banding pattern as the specific reaction product for future comparison.

\section{General Sliding-Strip Operation}

The sliding-strip devices enable the serial operations of sample preparation - including cell lysis, DNA isolation and purification - as well as LAMP amplification and detection. Unless otherwise noted, the procedure for sliding-strip assays, illustrated in Figure 4a, began with the application to the reaction disc of $10 \mu \mathrm{L}$ sample through the sample port. The strip was then slid to align the reaction disc with the wash port and $40 \mu \mathrm{L}$ of FTA Purification Buffer and $80 \mu \mathrm{L}$ of nuclease-free water were sequentially applied and allowed to transit through the disc to the paper vias and finally to the removable wash pad. The disc was then dried completely the strip slid to align the disc with the amplification reagent port, and $10 \mu \mathrm{L}$ of LAMP Master Mix was applied. The strip was then slid again, sealing the disc (by the magnetic force and the lubricant film) within the amplification zone. The device was placed in an incubator at $65^{\circ} \mathrm{C}$. After a 60 min incubation time, the devices were removed from the incubator and the strip was slid to the detection window, and the disc was dried completely. For detection, $10 \mu \mathrm{L}$ of $100 \mathrm{X}$ SYBR Green I in $1 \mathrm{X}$ TE was applied to the disc and it was excited using a handheld shortwave UV source and imaged using a camera phone. Images were then processed, converted to grayscale, and analyzed using Image $\mathrm{J}$ to quantify mean pixel intensity as gray value. The mean of the NTC replicates plus 3 times their standard deviation was used as the LOD, and the threshold value, for determining positive and negative reactions. In some cases, for ease of imaging and data 
presentation of multiple samples and replicates, the reaction discs were removed from the device and arrayed before imaging.

\section{Determination of Analytical Sensitivity}

The analytical sensitivity of amplification and detection in the sliding-strip device was determined using a double-stranded $200 \mathrm{bp}$ analog of the malB target sequence produced by Integrated DNA Technologies, Inc (Coralville, IA) as two single-stranded DNA Ultramers ${ }^{\circledR}$, the forward strand and the reverse complement. These strands were then combined to yield an equimolar solution, heated to $95^{\circ} \mathrm{C}$ for $5 \mathrm{~min}$ and slowly cooled to room temperature to anneal. The concentration was confirmed by spectrometry using the absorbance at $280 \mathrm{~nm}$. Serial dilutions were prepared, and $10 \mu \mathrm{L}$ applied to dry reaction discs, which had been pretreated with $10 \mu \mathrm{L}$ human plasma, $40 \mu \mathrm{L}$ of FTA Purification Buffer, and $80 \mu \mathrm{L}$ of nuclease-free water, through the amplification reagent port of the sliding-strip devices. The discs were then dried completely, and the general sliding-strip operations outlined above were followed starting from the application of LAMP Master Mix.

\section{Integrated Assay with Spiked Plasma Samples}

To examine the effectiveness of the sliding-strip device for sample preparation from complex matrices, the assay was challenged with samples of human plasma spiked with whole, live E. coli prepared from overnight culture as described above. All steps in the general slidingstrip operation outlined previously were followed. Here, parameters such as the number of washes, wash volumes, and wash buffer composition were optimized as shown Figure S2.

\section{Collecting and Concentrating DNA from Large Sample Volumes}


To examine the ability to isolate DNA from larger volumes of dilute samples, we applied $10,20,30,40$ or $50 \mu \mathrm{L}$ of a 0.05 cell $/ \mu \mathrm{L}$ sample of $E$. coli in human plasma to the reaction discs. After each sample wicked through the reaction disc, the general sliding-strip operation procedure was followed as described.

\section{Results \& Discussion}

\section{Fabrication of Sliding-Strip Devices with Evaporation-Resistant Dynamic Seals}

Nucleic acid-based diagnostic tests require serial operations to be performed on a complex sample in order to yield reliable results. DNA must be released from cells, isolated from the cell debris, proteins, and other sample components, purified of these potential polymerase inhibitors, amplified, and detected. Our device is designed to perform these operations typically done using laboratory equipment - with minimal user steps and at a fraction of the cost. The core architecture of the sliding-strip device, illustrated in Figure $1 \mathrm{a}$ and $\mathrm{b}$, consists of three major layers: the top layer, which provides ports to access a reaction disc; the middle layer, which consists primarily of the reaction disc housed in the sliding strip that is linearly actuated to serially address the ports; and the bottom layer, which contains paper vias to contact the reaction disc and the wash pad to transit waste wash out of the device. Each of these layers is fabricated out of a low-cost, flexible magnetic substrate, the attractive force of which, when combined with the thin film of lubricant, creates a dynamic seal that allows for extended incubation at $65{ }^{\circ} \mathrm{C}$ with negligible evaporation (Figure 2). As shown in Figure 1c, these devices are small - only $0.225 \mathrm{~cm}$ thick and the prototype device, capable of running one sample and two controls, is 4.7 $\mathrm{cm}$ wide and $10 \mathrm{~cm}$ from the top to the end of the strip-pull when it is in the starting position and will ultimately include an inexpensive electronic heater. This, in combination with on-board storage of lyophilized reagents, enables point-of-care use. 


\section{Analytical Sensitivity in Paper}

To assess the performance of the LAMP reaction when conducted in buffer contained within a paper disc that is housed in the sliding strip, we examined serial dilutions of the $200 \mathrm{bp}$ dsDNA target sequence in nuclease-free water. By drying these samples onto reaction discs, which had been treated with human plasma and washed, we replicated the condition of the discs that would exist in the full assay prior to amplification and isolated potential amplification inhibition from sample preparation loses, both of which could yield false negative results. As shown in Figure 3a, the reaction proceeds uninhibited and reactions starting with as few as 1 copy yield a statistically significant signal over the no template control (NTC) $(n=8, p<1 E-11)$. As is evident in Figure 3b, a camera phone image of the reaction discs under UV excitation, these optimal conditions and idealized sample produce a binary result - common with endpoint detection of isothermal amplification reactions.

Though no sequence homology exists between primers in the set, in sub-optimal conditions mismatches may be tolerated. To confirm that fluorescent signal is the result of the specific template-driven reaction, a restriction endonuclease analysis was performed as described in the supporting information and shown in Figure S4.

\section{Integrated Assay with Spiked Plasma Samples}

The assay integrated with the sliding-strip device, was challenged with whole, live E. coli serially diluted in human plasma. The samples were subjected to all of the steps of sample preparation, in order as described in the Materials \& Methods section, by linear actuation of the sliding strip to the appropriate ports for application of the wash buffer, LAMP Master Mix, and detection reagents, as detailed in Figure 4a. Upon imaging, positive results were obtained from four of six samples containing as few as 1 cell (Figure 4b), this outcome is consistent with 
stochastic sample error as predicted by the most probable number (MPN) method (described in the supporting information).

As shown in Figure S5, statistical significance $(\mathrm{p}<0.001)$ is achieved for all concentrations over 5 cells per sample (e.g. 0.5 cells $/ \mu \mathrm{L}$ or 500 cells $/ \mathrm{mL}$ ).

\section{Collecting and Concentrating DNA from Larger Sample Volumes}

Our intended use of a product based on this system includes direct application of $30 \mu \mathrm{L}$ of finger-stick blood, the average volume obtained from a safety lancet. As the limit of detection of 500 cells $/ \mathrm{mL}$ was achieved using a $10 \mu \mathrm{L}$ sample volume, we investigated the effects of larger sample volumes. By varying the volume of a sample containing 0.05 cells $/ \mu \mathrm{L}$ (an order of magnitude lower than the above established limit of detection) in increments of $10 \mu \mathrm{L}$ applied to different reaction discs, as shown in Figure 5, we noted an increase in the number of replicates resulting in positive amplification with 10 and $20 \mu \mathrm{L}$ volumes (approximately 0.5 and 1 cell/disc, respectively) producing no positive signals, $40 \mu \mathrm{L}$ (approximately 2 cells/disc) producing 7 positive replicates, and $60 \mu \mathrm{L}$ (approximately 3 cells/disc) yielding positive signals in all 8 replicates. This demonstrates that the additional cells present in the larger volume samples are being successfully lysed and their DNA captured by the cellulose fibers of the paper reaction discs. This concentration effect is limited. Volumes over $60 \mu \mathrm{L}$ degrade these gains, with 80 and $100 \mu \mathrm{L}$ volumes (approximately 4 and 5 cells/disc, respectively) dropping to 5 and 3 positive replicates, respectively. This decline is likely due to washing effects; both washing away of the dried lysis chemistry of the FTA ${ }^{\circledR}$ paper disc, preventing the release of additional target, and washing away of previously captured target.

\section{Conclusions}


We designed, fabricated, and tested an inexpensive technology for molecular diagnostics that enables sample preparation steps of cell lysis, DNA isolation, concentration, and purification integrated with LAMP amplification and detection. The basis for fabrication uses a patterned strip that contains the reaction mixture in a paper disc, and slides this disc from station to station to accomplish addition of sample, lysis of cells (if relevant), adsorption of DNA, washing, and addition of reagents. This simple sliding motion of the strip in the "paper machine," in conjunction with the ability of paper to cause the transport of water by capillary wicking, replaces most of the equipment needed to conduct NAATs on the benchtop - centrifuges, vortex mixers - and, in a consumer product, pipettors. The assembly is held together using synthetic rubber-bonded ferrite magnets and laminates. The compressive force of the magnets on the strip and thin film of lubricant between the layers creates a dynamic seal that resists evaporation, and allows for incubation of the amplification reaction at $65^{\circ} \mathrm{C}$ for 1 hour.

Using a dsDNA analog of the target region within the malB gene of E. coli, we demonstrated the LAMP reaction can be run within the cellulose fiber matrix of a paper disc with an analytical sensitivity of a single copy of the target sequence. When integrated with the entire procedure for sample preparation, and beginning from whole, live E. coli cells in human plasma, we were able to detect consistently, 5 cells, and presence of the organism was detected on 4 out of 6 samples containing 1 cell.

We also demonstrated the ability to concentrate target in the reaction disc by applying larger sample volumes. This may permit less precise metering of sample in a product based on this system (e.g. direct application of a finger-stick blood drop), making it more user friendly and eliminating the need for pipettors. Further, the use of larger sample volumes may improve 
detection at low concentrations by increasing the probability of capturing the target in the sample volume.

The current prototype uses an inexpensive detection scheme - excitation of an intercalating fluorophore with a UV source and camera-phone-based imaging - that can be adapted directly to a marketable product. This system replaces complicated and expensive optics, such as those found in a fluorimeter. The requirement of even this simple equipment, and the incubator required to heat the device, may limit use in some environments. To address this, current work is focused on replacing this detection scheme with one that produces a colorimetric signal visible with the unaided eye in ambient light. Similarly, we are designing and testing a low-cost, electronic heater built-in to the device to eliminate the incubator.

The use of liquid amplification reagents in this proof-of-concept will prevent application in resource-limited environments as they have a limited shelf-life in the absence of refrigeration. Ongoing work is focused on lyophilizing these reagents on-board to address this problem.

A key metric in the development of this device is cost; in order for a product based on this system to be widely adopted in resource-limited countries it must be affordable. This "paper machine," as presented, is low-cost through its use of paper microfluidics and an isothermal amplification reaction with endpoint detection. Through continued development of this system, we envision a a total-disposable, non-instrumented device that can dramatically reduce the cost of nucleic acid diagnostic tests for infectious diseases, genetic conditions, and cancer and bring it to the point-of-care in resource-limited settings.

\section{Acknowledgements}

This work was supported by the Defense Advanced Research Projects Agency (Grant Number HR0011-12-2-0010). 


\section{References}

(1) Al-Soud, W. A.; Rådström, P. J. Clin. Microbiol. 2001, 39, 485-493.

(2) Wilson, I. G. Appl. Environ. Microbiol. 1997, 63, 3741-3751.

(3) Cordray, M. S.; Richards-Kortum, R. R. Am. J. Trop. Med. Hyg. 2012, 87, 223-230.

(4) LaBarre, P.; Hawkins, K. R.; Gerlach, J.; Wilmoth, J.; Beddoe, A.; Singleton, J.; Boyle, D.; Weigl, B. PLoS One 2011, 6, e19738.

(5) Mabey, D.; Peeling, R. W.; Ustianowski, A.; Perkins, M. D. Nat. Rev. Micro. 2004, 2, 231240.

(6) Weigl, B. H.; Boyle, D. S.; Santos, T. d. 1.; Peck, R. B.; Steele, M. S. Expert Rev. Med. Devices 2009, 6, 461-464.

(7) Barfield, C. A.; Barney, R. S.; Crudder, C. H.; Wilmoth, J. L.; Stevens, D. S.; Mora-Garcia, S.; Yanovsky, M. J.; Weigl, B. H.; Yanovsky, J. IEEE Trans. Biomed. Eng. 2011, 58, 814-817.

(8) Engler, K. H.; Efstratiou, A.; Norn, D.; Kozlov, R. S.; Selga, I.; Glushkevich, T. G.; Tam, M.; Melnikov, V. G.; Mazurova, I. K.; Kim, V. E.; Tseneva, G. Y.; Titov, L. P.; George, R. C. J. Clin. Microbiol. 2002, 40, 80-83.

(9) Labbé, A. C.; Pillai, D. R.; Hongvangthong, B.; Vanisaveth, V.; Pomphida, S.; Inkathone, S.; Hay Burgess, D. C.; Kain, K. C. Ann. Trop. Med. Parasitol. 2001, 95, 671-677.

(10) Patterson, K.; Olsen, B.; Thomas, C.; Norn, D.; Tam, M.; Elkins, C. J. Clin. Microbiol. 2002, 40, 3694-3702.

(11) Aveyard, J.; Mehrabi, M.; Cossins, A.; Braven, H.; Wilson, R. Chem. Commun. 2007, 42514253.

(12) Connelly, J. T.; Nugen, S. R.; Borejsza-Wysocki, W.; Durst, R. A.; Montagna, R. A.; Baeumner, A. J. Anal. Bioanal. Chem. 2008, 391, 487-495. 
(13) Soliman, H.; El-Matbouli, M. Mol. Cell. Probes 2010, 24, 38-43.

(14) Mens, P. F.; van Amerongen, A.; Sawa, P.; Kager, P. A.; Schallig, H. D. F. H. Diagn. Microbiol. Infect. Dis. 2008, 61, 421-427.

(15) Chen, D.; Mauk, M.; Qiu, X.; Liu, C.; Kim, J.; Ramprasad, S.; Ongagna, S.; Abrams, W. R.; Malamud, D.; Corstjens, P. L.; Bau, H. H. Biomed. Microdevices 2010, 12, 705-719.

(16) Chen, Z.; Mauk, M. G.; Wang, J.; Abrams, W. R.; Corstjens, P. L. A. M.; Niedbala, R. S.; Malamud, D.; Bau, H. H. Ann. N. Y. Acad. Sci. 2007, 1098, 429-436.

(17) Roskos, K.; Hickerson, A. I.; Lu, H.-W.; Ferguson, T. M.; Shinde, D. N.; Klaue, Y.; Niemz, A. PLoS One 2013, 8, e69355.

(18) Boehme, C. C.; Nabeta, P.; Hillemann, D.; Nicol, M. P.; Shenai, S.; Krapp, F.; Allen, J.;

Tahirli, R.; Blakemore, R.; Rustomjee, R.; Milovic, A.; Jones, M.; O'Brien, S. M.; Persing, D. H.; Ruesch-Gerdes, S.; Gotuzzo, E.; Rodrigues, C.; Alland, D.; Perkins, M. D. N. Engl. J. Med. 2010, 363, 1005-1015.

(19) Evans, C. A. PLoS Med. 2011, 8, e1001064.

(20) Nagamine, K.; Hase, T.; Notomi, T. Mol. Cell. Probes 2002, 16, 223-229.

(21) Notomi, T.; Okayama, H.; Masubuchi, H.; Yonekawa, T.; Watanabe, K.; Amino, N.; Hase, T. Nucleic Acids Res. 2000, 28, e63.

(22) Ihira, M.; Akimoto, S.; Miyake, F.; Fujita, A.; Sugata, K.; Suga, S.; Ohashi, M.; Nishimura, N.; Ozaki, T.; Asano, Y.; Yoshikawa, T. J. Clin. Virol. 2007, 39, 22-26.

(23) Iseki, H.; Kawai, S.; Takahashi, N.; Hirai, M.; Tanabe, K.; Yokoyama, N.; Igarashi, I. J. Clin. Microbiol. 2011, 48, 2509-2514.

(24) Kaneko, H.; Kawana, T.; Fukushima, E.; Suzutani, T. J. Biochem. Biophys. Methods 2007, $70,499-501$. 
(25) Wang, F.; Jiang, L.; Ge, B. J. Clin. Microbiol. 2012, 50, 91-97.

(26) Boehme, C. C.; Nabeta, P.; Henostroza, G.; Raqib, R.; Rahim, Z.; Gerhardt, M.; Sanga, E.;

Hoelscher, M.; Notomi, T.; Hase, T.; Perkins, M. D. J. Clin. Microbiol. 2007, 45, 1936-1940.

(27) Boyanton, B. L.; Sural, P.; Loomis, C. R.; Pesta, C.; Gonzalez-Krellwitz, L.; Robinson-

Dunn, B.; Riska, P. J. Clin. Microbiol. 2011, 50, 640-645.

(28) Carrilho, E.; Martinez, A. W.; Whitesides, G. M. Anal. Chem. 2009, 81, 7091-7095.

(29) Martinez, A. W.; Phillips, S. T.; Butte, M. J.; Whitesides, G. M. Angew. Chem. Int. Ed.

Engl. 2007, 46, 1318-1320.

(30) Martinez, A. W.; Phillips, S. T.; Whitesides, G. M. Proc. Natl. Acad. Sci. U. S. A. 2008, 105, 19606-19611.

(31) Martinez, A. W.; Phillips, S. T.; Whitesides, G. M.; Carrilho, E. Anal. Chem. 2009, 82, 310.

(32) Fu, E.; Lutz, B.; Kauffman, P.; Yager, P. Lab Chip 2010, 10, 918-920.

(33) Lutz, B. R.; Trinh, P.; Ball, C.; Fu, E.; Yager, P. Lab Chip 2011, 11, 4274-4278.

(34) Osborn, J. L.; Lutz, B.; Fu, E.; Kauffman, P.; Stevens, D. Y.; Yager, P. Lab Chip 2010, 10, 2659-2665.

(35) Alberts, B. M.; Amodio, F. J.; Jenkins, M.; Gutmann, E. D.; Ferris, F. L. Cold Spring Harb. Symp. Quant. Biol. 1968, 33, 289-305.

(36) Yetisen, A. K.; Akram, M. S.; Lowe, C. R. Lab Chip 2013, 13, 2210-2251.

(37) Pollock, N. R.; Rolland, J. P.; Kumar, S.; Beattie, P. D.; Jain, S.; Noubary, F.; Wong, V. L.;

Pohlmann, R. A.; Ryan, U. S.; Whitesides, G. M. Sci. Transl. Med. 2012, 4, 152ra129. 
(38) Pollock, N. R.; McGray, S.; Colby, D. J.; Noubary, F.; Nguyen, H.; Nguyen, T. A.;

Khormaee, S.; Jain, S.; Hawkins, K.; Kumar, S.; Rolland, J. P.; Beattie, P. D.; Chau, N. V.;

Quang, V. M.; Barfield, C.; Tietje, K.; Steele, M.; Weigl, B. H. PLoS One 2013, 8, e75616.

(39) Rohrman, B. A.; Richards-Kortum, R. R. Lab Chip 2012, 12, 3082-3088.

(40) Allen, P. B.; Arshad, S. A.; Li, B.; Chen, X.; Ellington, A. D. Lab Chip 2012, 12, 2951 2958.

(41) Li, C.-C.; Beck, I. A.; Seidel, K. D.; Frenkel, L. M. J. Clin. Microbiol. 2004, 42, 3847-3849.

(42) Picard-Meyer, E.; Barrat, J.; Cliquet, F. J. Virol. Methods 2007, 140, 174-182.

(43) Turse, J. E.; Pei, J.; Ficht, T. A. Front. Microbiol. 2011, 2, 54-66.

(44) Hill, J.; Beriwal, S.; Chandra, I.; Paul, V. K.; Kapil, A.; Singh, T.; Wadowsky, R. M.; Singh, V.; Goyal, A.; Jahnukainen, T.; Johnson, J. R.; Tarr, P. I.; Vats, A. J. Clin. Microbiol. 2008, 46, $2800-2804$.

(45) Tanner, N. A.; Zhang, Y.; Evans, T. C., Jr. Biotechniques 2012, 53, 81-89. 
(a.) Exploded Device

\section{(b.) Assembled Device}
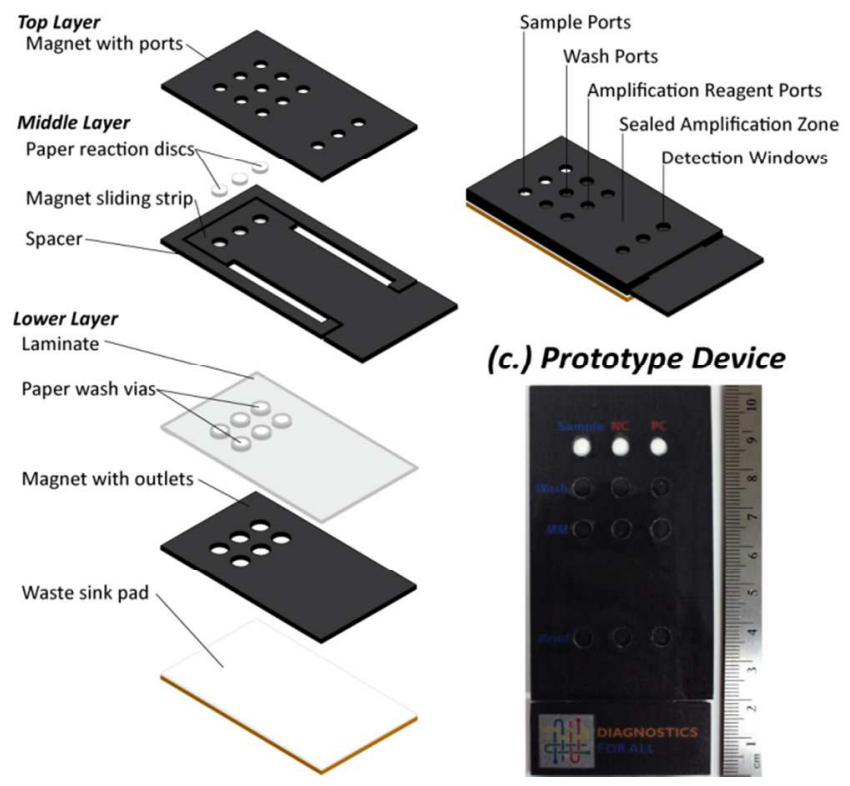

(c.) Prototype Device

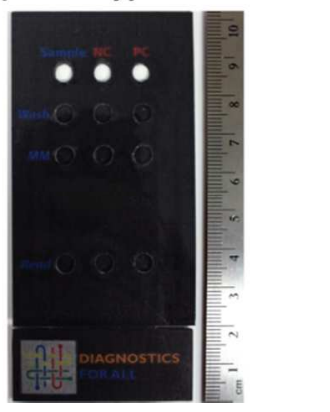

Figure 1: Schematic of Sliding-Strip Device. The exploded view (a) shows the three major layers of the sliding-strip architecture and their components. The assembled device (b) can accommodate a single sample and controls. A prototype device (c) contains three reactions discs - one sample and a negative (NC) and positive (PC) control - with ports to add the samples, wash buffer, amplification Master Mix (MM) and to add detection reagents and visual the signal (Read). 


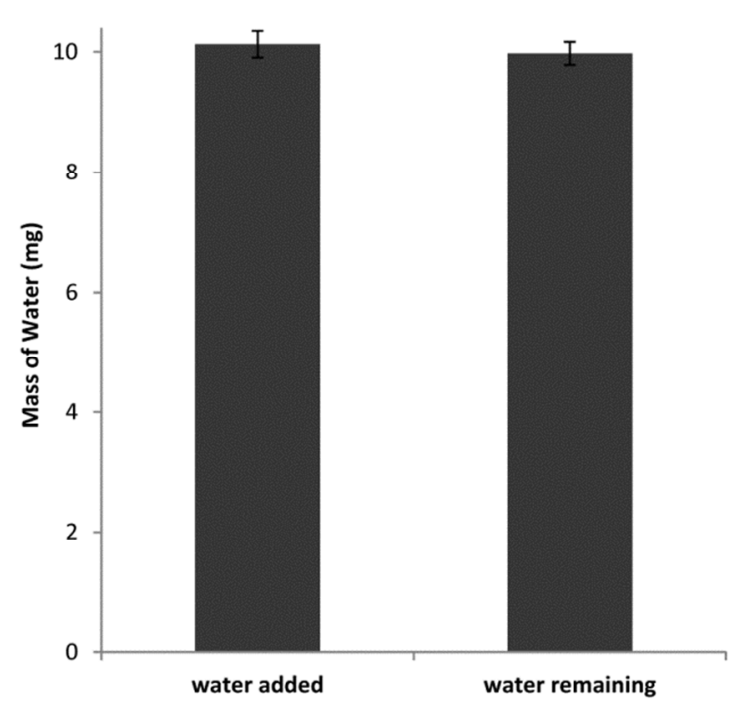

Figure 2: Demonstration of Evaporation-Resistance. The magnetic substrate used to fabricate the device, in conjunction with the thin film oflubricant, creates an evaporation-resistant seal, with an average loss of less than $0.02 \mu \mathrm{L}$ of $10 \mu \mathrm{L}$ water applied $(\mathrm{n}=4)$ after 1 hour of heating at $65^{\circ} \mathrm{C}$. 


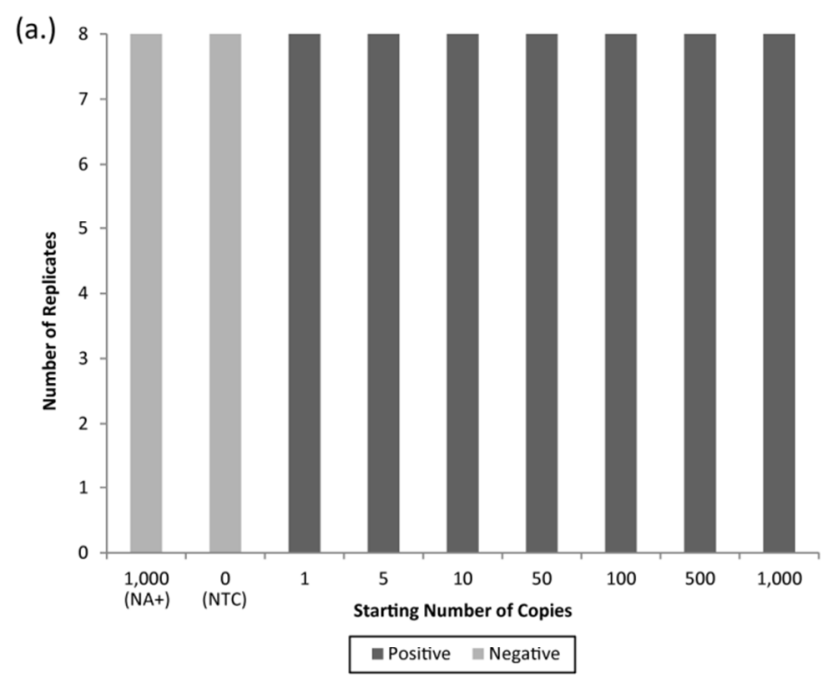

(b.)

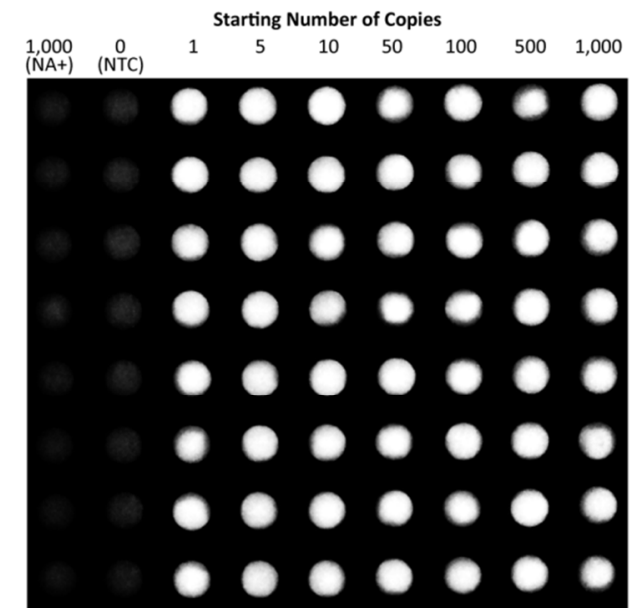

Figure 3: Analytical Sensitivity of LAMP in Sliding-Strip Device. All reactions containing as few as 1 copy of the 200 bp dsDNA target yielded detectable amplification (a). Positive replicates are those producing signals greater than the limit of detection (LOD) calculated as the average plus three times the standard deviation of the no template control (NTC) $(n=8)$. Average signal data is presented in Figure S3. The data is generated by photographing the discs under UV excitation (b) after application of $10 \mu \mathrm{L}$ of 100x SYBR Green I and quantifying the mean gray values with ImageJ. 
(a.) Cross section of Sliding Strip

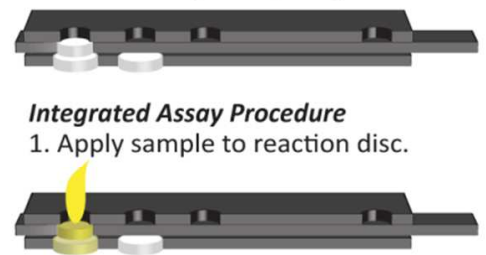

2. Slide strip to move reaction disc to wash port. Apply wash buffers to reaction disc.

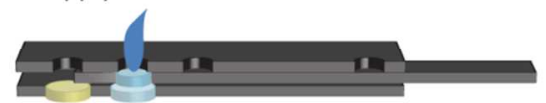

3. Slide strip to move reaction disc to regeant port. Apply Master Mix to reaction disc.

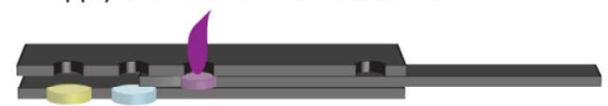

4. Slide strip to sealed amplification zone and incubate.

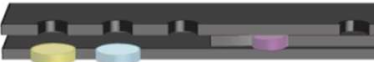

5. Slide stripto move reaction disc to detection window. Apply SYBR Green I, excite with UV, and image.

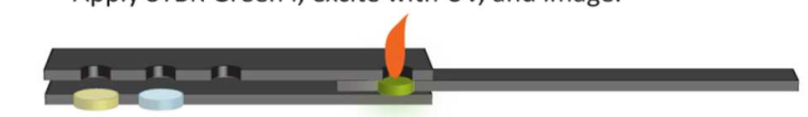

(b.) 6

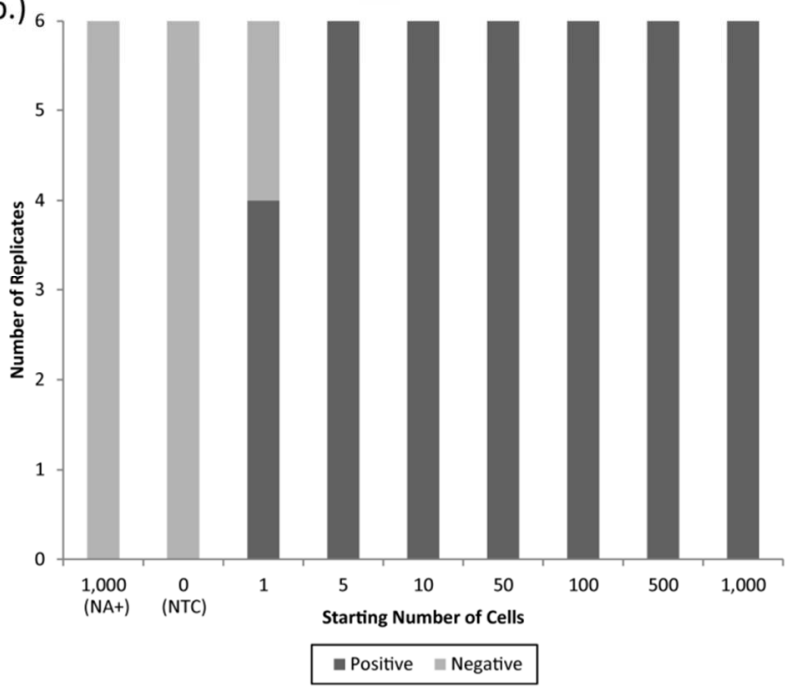

Figure 4: Assay Integrated with Sliding-Strip Device using Spiked Plasma. The entire

bioanalytical assay, from sample preparation through amplification and detection, was integrated with the sliding-strip devices. Visualized in cross-section (a), the sample consisting of whole, live E. coli spiked into human plasma was applied to the reaction disc, and then washing, reagent addition, incubation, and detection were controlled in series via linear actuation of the sliding 
strip. As before, the replicates were binned as positive and negative (b), based on mean gray values quantified via ImageJ (Figure S5) $(n=6)$. 


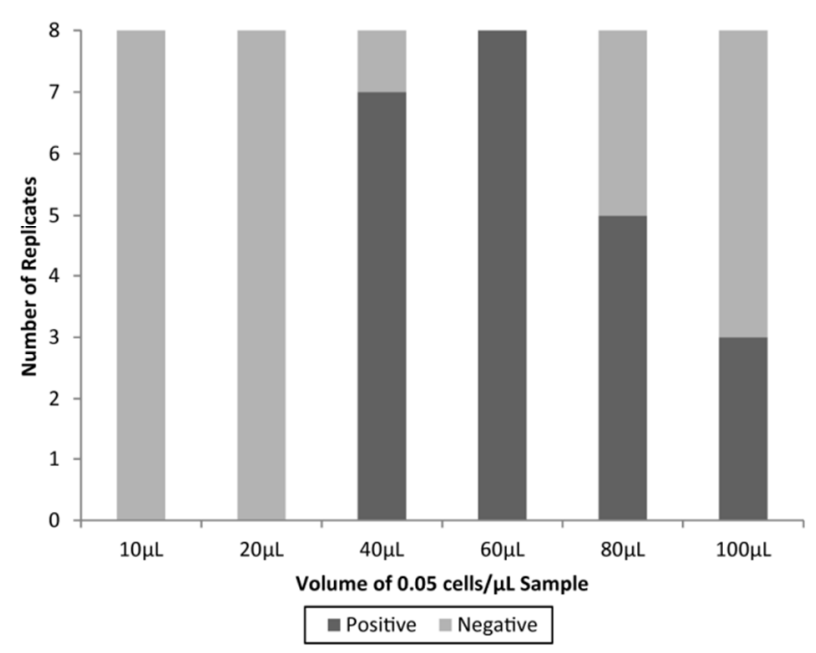

Figure 5: Collection and Concentration of Target from Larger Sample Volumes. Samples of 0.05 cells $/ \mu \mathrm{L} E$. coli in human plasma were applied in $10 \mu \mathrm{L}$ increments to reactions discs in sliding-strip devices and processed through the integrated sample preparation, amplification and detection procedures of the device $(n=8)$. Signals from all replicates of 10 and $20 \mu \mathrm{L}(\sim 0.5$ and 1 cell applied to discs) samples remained below the LOD, determined as the mean plus 3 times the standard deviation of a NTC run in parallel, and were thus scored as negative. With 40 and $60 \mu \mathrm{L}$ samples ( 2 and 3 cells) the number of replicates scoring positive, above the LOD, increased to 7 and 8 , respectively. Sample volumes over $60 \mu \mathrm{L}$ began to reduce the number of replicates positive likely as a result of washing effects. Average signal and average positive signal data is shown in Figure S6. 